\title{
Planetary Dynamics in the $\alpha$ Centauri System: Lyapunov Spectra and Long-term Behaviour
}

\author{
E. A. Popova and I. I. Shevchenko \\ Pulkovo Observatory of the Russian Academy of Sciences \\ Pulkovskoye ave. 65, Saint Petersburg 196140, Russia \\ email: m02pea@hotmail.com
}

\begin{abstract}
The stability of planetary motion in the binary system $\alpha$ Cen A-B is studied. Lyapunov spectra of the motion of the system with a single massive planet are computed on a fine grid of the initial data, and, by means of statistical analysis of the obtained data arrays, chaotic domains are identified in the "pericentric distance - eccentricity" initial data space for the planetary orbit. Association with the initial data domains for the orbits exhibiting close encounters with central stars and for the orbits exhibiting long-term escape is investigated.
\end{abstract}

Keywords. Celestial mechanics, planetary systems, methods: numerical

The orbital dynamics of hypothetical planets in the $\alpha$ Cen system was extensively studied by Benest (1988), Benest (1989), Wiegert \& Holman (1997), Wiegert \& Holman (1999), Benest \& Gonczi (1998), Benest \& Gonczi (2003), and other authors. We consider the double star $\alpha$ Cen A-B, setting the component masses $M_{1}=1.1 M_{\odot}, M_{2}=0.91 M_{\odot}$, semi-major axis $a=23.4 \mathrm{AU}$ and eccentricity $e=0.52$ (Pourbaix et al. 1999). We perform computations of the planetary orbits in the two cases: the planar elliptic restricted threebody problem (the planet mass is zero) and the planar general three-body problem (the planet mass is nonzero; it is set equal to one Jupiter mass). At the initial moment of time, the three bodies are located on the "B-A-planet" straight line at the pericenters of their unperturbed orbits. We integrate on a fine grid of the initial data for the planet orbit, varying the pericentric distance $q$ and the eccentricity $e$.

To explore the stability problem for the planetary motion, we use two stability criteria. The first criterion is the "escape-collision" one: the orbit is stable if the distance between the planet and one of the stars does not become less than $10^{-3}$ AU and does not exceed $10^{3} \mathrm{AU}$. The second criterion is the value of the maximum Lyapunov characteristic exponent (maximum LCE). We perform the computations of the full LCE spectra using the algorithms and codes by von Bremen et al. (1997), Shevchenko \& Kouprianov (2002), Kouprianov \& Shevchenko (2003), Kouprianov \& Shevchenko (2005). The LCE spectra are obtained for each point on the grid of the initial data.

The statistical method, used here for the separation of regular and chaotic orbits, was proposed by Melnikov \& Shevchenko (1998) (see also Shevchenko 2002, Shevchenko \& Melnikov 2003). It consists of 4 steps. (i) Two differential distributions of the maximum LCE are constructed for the initial data grid using two different integration time intervals. (ii) Each of these distributions has at least two peaks. The peak that shifts (moves in the direction of the LCE smaller values), when the integration time interval is increased, corresponds to the regular trajectories. The fixed peak (peaks) corresponds to the chaotic ones. (iii) The maximum LCE value in the middle between the peaks (in the distribution built taking the maximum computation time) gives the numerical 


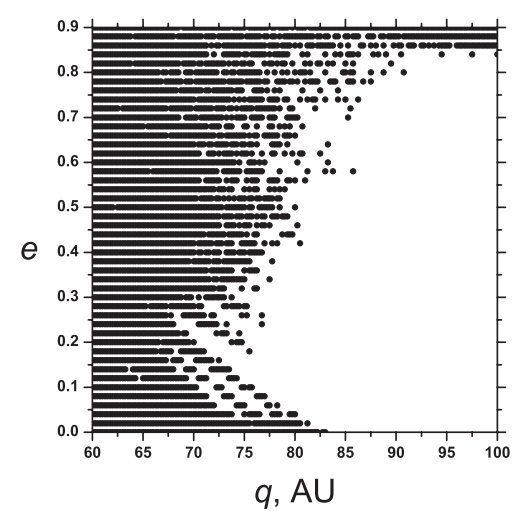

a)

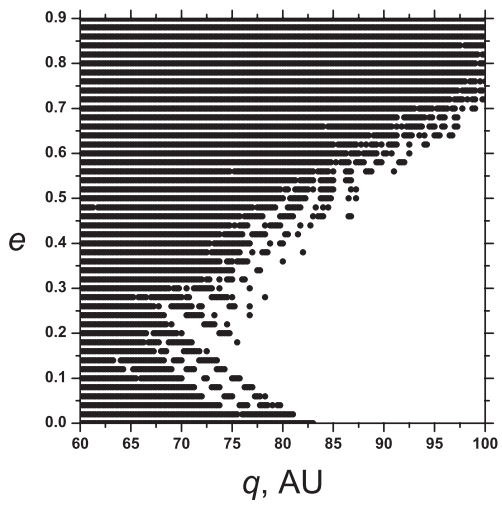

b)

Figure 1. Stability diagrams constructed by the "escape-collision" (a) and LCE (b) criteria.

criterion for the separation of the regular and chaotic trajectories. (iv) In the further computations on finer data grids, the obtained criterion is used to separate the regular and chaotic trajectories using much smaller time intervals of integration.

Examples of the constructed stability diagrams are shown in Fig. 1. These are two diagrams for the outer orbits of the system in the general three-body problem. Fig. $1 a$ is constructed by the "escape-collision" criterion, and Fig. $1 b$ by the maximum LCE criterion. The chaotic domains are shown in black.

Our basic conclusions are as following. (i) For the initially circular planetary orbits, the outer borders of the chaotic domains in the stability diagrams correspond to the semimajor axis $\sim 80 \mathrm{AU}$, whereas the inner borders correspond to $\sim 5 \mathrm{AU}$. (ii) The chaotic domains in the diagrams expand approximately linearly with the eccentricity. (iii) The representative values of the Lyapunov times in the chaotic domains are: $\sim 500 \mathrm{yr}$ for the outer orbits, and $\sim 60 \mathrm{yr}$ for the inner orbits. $(i v)$ The LCE criterion, applied for the construction of the stability diagrams, provides a better resolution of the chaos-order borders in the stability diagrams in comparison with the "escape-collision" criterion.

\section{References}

Benest, D. 1988, A\&SA, 206, 143

Benest, D. 1989, A\&A A, 223, 361

Benest, D. \& Gonczi, R. 1998, Earth, Moon, and Planets, 81, 7

Benest, D. \& Gonczi, R. 2003, Earth, Moon and Planets, 93, 175

von Bremen, H. F., Udwadia, F. E., \& Proskurowski, W. 1997, Physica D, 101, 1

Kouprianov, V. V. \& Shevchenko, I. I. 2003, A\&A, 410, 749

Kouprianov, V. V. \& Shevchenko, I. I. 2005, Icarus, 176, 224

Melnikov, A. V. \& Shevchenko, I. I. 1998, Sol. Sys. Res., 32, 480 [Astron. Vestnik, 32, 548]

Pourbaix, D., Neuforge-Verheecke, C., \& Noels, A. 1999, A\& A, 344, 172

Shevchenko, I. I. 2002, in: Asteroids, Comets, Meteors 2002. Ed. by Warmbein B., Berlin: ESA, 2002. P. 367-370.

Shevchenko, I. I. \& Kouprianov, V. V. 2002, A\&A, 394, 663

Shevchenko, I. I. \& Melnikov, A. V. 2003, JETP Lett., 77, 642 [Pis'ma ZhETF, 77, 772]

Wiegert, P. A. \& Holman, M. J. 1997, AJ, 113, 1445

Wiegert, P. A. \& Holman, M. J. 1999, AJ, 117, 621 